\title{
Numerical Solution for Third-Order Two-Point Boundary Value Problems with the Barycentric Rational Interpolation Collocation Method
}

\author{
Qian Ge $\mathbb{D}$ and Xiaoping Zhang $\mathbb{1}$ \\ School of Science, Shandong Jianzhu University, Jinan 250101, China \\ Correspondence should be addressed to Xiaoping Zhang; 15098931339@163.com
}

Received 19 November 2020; Revised 26 January 2021; Accepted 4 February 2021; Published 18 February 2021

Academic Editor: Jin Li

Copyright (C) 2021 Qian Ge and Xiaoping Zhang. This is an open access article distributed under the Creative Commons Attribution License, which permits unrestricted use, distribution, and reproduction in any medium, provided the original work is properly cited.

\begin{abstract}
The numerical solution for a kind of third-order boundary value problems is discussed. With the barycentric rational interpolation collocation method, the matrix form of the third-order two-point boundary value problem is obtained, and the convergence and error analysis are obtained. In addition, some numerical examples are reported to confirm the theoretical analysis.
\end{abstract}

\section{Introduction}

Differential equations can give full play to their mathematical advantages in various disciplines. Combining the theory of differential equations with practical problems can build models of practical problems. Many engineering and physical problems can be transformed into the initial boundary value problems of differential equations. In these problems, only a few simple cases can be solved analytically, and most engineering problems need to be solved by numerical methods. Compared with polynomial interpolation, rational function interpolation has higher interpolation accuracy and can effectively overcome the instability of interpolation [1-4]. Barycentric rational interpolation not only has high interpolation accuracy on special distributed nodes but also has high interpolation accuracy for equidistant nodes [5-7]. This method has been used to solve certain problems such as Volterra integral equations [2, 8, 9], delay Volterra integrodifferential equations $[10,11]$, plane elastic problems [12], nonlinear problems [13], heat conduction equation [14], and so on [15-17].

The third-order differential equation has a wide range of applications and important theoretical values in many scientific fields, such as applied mathematics and physics. Therefore, the third-order boundary value problem has been widely concerned by many scholars [18-20]. In this paper, we consider the numerical solution of the third-order twopoint boundary value problem,

$$
\begin{gathered}
u^{\prime \prime \prime}(x)+p u \prime \prime(x)+q u^{\prime}(x)+r u(x)=f(x), \quad a<x<b, \\
u(a)=A, u^{\prime}(a)=B, u^{\prime}(b)=C, \text { (or) } \\
u(a)=A, u^{\prime}(a)=B, u{ }^{\prime \prime}(a)=C,
\end{gathered}
$$

by the barycentric rational interpolation collocation method.

Barycentric rational interpolation collocation method means using barycentric interpolation polynomials to find the differential matrix of a function at each discrete point; thus, the solution of the differential equation can be obtained by matrix operation. The barycentric rational interpolation has excellent numerical stability and high approximation accuracy, and the barycentric rational interpolation formula has a compact calculation formula of all order derivatives. Therefore, the barycentric rational interpolation collocation 
method is an effective method for solving boundary value problems of differential equations.

\section{Formula of the Barycentric Interpolation Collocation Method}

Discretize the interval $[a, b]$ into $n$ uniform parts with $h=((b-a) / n)$, and suppose $u_{1}, u_{2}, \ldots, u_{n}$ is the function value of an unknown function $u$ at discrete nodes $x_{1}, x_{2}, \ldots, x_{n}$.

For any $0 \leq d \leq n, P\left(x_{i}\right), i=0,1, \ldots, n-d$, is the interpolation function at the point $x_{i}, x_{i+1}, \ldots, x_{i+d}$; then, we have $P_{i}\left(x_{k}\right)=f\left(x_{k}\right), k=i, i+1, \ldots, i+d$, and

$$
r(x)=\frac{\sum_{i=0}^{n-d} \lambda_{i}(x) P_{i}(x)}{\sum_{i=0}^{n-d} \lambda_{i}(x)}
$$

where

$$
\lambda_{i}(x)=\frac{(-1)^{i}}{\left(x-x_{i}\right) \cdots\left(x-x_{i+d}\right)} .
$$

By changing the polynomial $P_{i}(x)$ into the Lagrange interpolation form as

$$
P_{i}(x)=\sum_{k=i}^{i+d} \prod_{j=i, j \neq k}^{i+d} \frac{x-x_{j}}{x_{k}-x_{j}} f_{k}
$$

and combining (4) and (5) together, we get

$$
\sum_{i=0}^{n-d} \lambda_{i}(x) P_{i}(x)=\sum_{i=0}^{n-d}(-1)^{i} \sum_{k=i}^{i+d} \frac{1}{x-x_{k}} \prod_{j=i, j \neq k}^{i+d} \frac{1}{x_{k}-x_{j}} f_{k}=\sum_{k=0}^{n} \frac{w_{k}}{x-x_{k}} f_{k},
$$

where $w_{k}=\sum_{i \in J_{k}}(-1)^{i} \prod_{j=i, j \neq k}^{i+d}\left(1 \quad / x_{k}-x_{j}\right), J_{k}=\{i \in I ; k-$ $d \leq i \leq k\}$.

Then, we get

$$
r(x)=\frac{\sum_{j=0}^{n}\left(w_{j} / x-x_{j}\right) f_{j}}{\sum_{j=0}^{n}\left(w_{j} / x-x_{j}\right)}
$$

where its basis function is

$$
L_{j}(x)=\frac{\left(w_{j} / x-x_{j}\right)}{\sum_{k=0}^{n}\left(w_{k} / x-x_{k}\right)} .
$$

For the equidistant point, the weight function is

$$
w_{j}=(-1)^{n-j} C_{n}^{j}
$$

For the Chebyshev point of the second kind,

$$
x_{j}=\cos \frac{j \pi}{n}, \quad j=0,1, \ldots, n,
$$

the weight function is

$$
w_{j}=(-1)^{j} \delta_{j}, \delta_{j}= \begin{cases}\frac{1}{2}, & j=0, n, \\ 1, & \text { otherwise. }\end{cases}
$$

By formula (8), the $m$-th order derivative of $u(x)$ at the nodes $x_{1}, x_{2}, \ldots, x_{n}$ can be expressed as

$$
\begin{aligned}
u^{(m)}\left(x_{j}\right) & :=u_{i}^{(m)}=\frac{d^{m} u\left(x_{j}\right)}{\mathrm{d} x^{m}}=\sum_{k=1}^{n} L_{k}^{(m)}\left(x_{j}\right) u_{k} \\
& =\sum_{k=1}^{n} D_{i j}^{(m)} u_{k}, \quad m=1,2, \ldots,
\end{aligned}
$$

and then (12) can be written in the matrix form as

$$
u^{(m)}=D^{(m)} u \text {, }
$$

where $\quad u^{(m)}=\left[u_{1}^{(m)}, u_{2}^{(m)}, \ldots, u_{n}^{(m)}\right]^{T} \quad$ and $u=\left[u_{1}, u_{2}, \ldots, u_{n}\right]^{T}$.

By using the barycentric interpolation function as

$$
u_{n}(x)=\sum_{j=0}^{n} L_{j}(x) u_{j}
$$

equation (1) can be written in the numerical form as

$$
\sum_{j=0}^{n} u_{j} L_{j}^{\prime \prime}(x)+p \sum_{j=0}^{n} u_{j} L_{j}^{\prime \prime}(x)+q \sum_{j=0}^{n} u_{j} L_{j}^{\prime}(x)+r \sum_{j=0}^{n} u_{j} L_{j}(x)=f(x) .
$$


By using the notation of the differential matrix, (15) can also be denoted as

$$
\begin{aligned}
& \sum_{j=0}^{n} D_{i j}^{(3)} u_{j}+p \sum_{j=0}^{n} D_{i j}^{(2)} u_{j}+q \sum_{j=0}^{n} D_{i j}^{(1)} u_{j}+r \\
& \sum_{j=0}^{n} \delta_{i j} u_{j}=f\left(x_{i}\right), \quad i=1,2, \ldots, n,
\end{aligned}
$$

or the simple matrix form

$$
\left[D^{(3)}+p D^{(2)}+q D^{(1)}+r I\right] \mathbf{u}=\mathbf{f} .
$$

Boundary conditions (2) can be divided into

$$
\begin{aligned}
u_{1} & =A, u^{\prime}\left(x_{1}\right)=\sum_{j=0}^{n} D_{1 j}^{(1)} u_{j}=B, \\
u^{\prime}\left(x_{n}\right) & =\sum_{j=0}^{n} D_{n j}^{(1)} u_{j}=C, \text { (or) } \\
u_{1} & =A, u^{\prime}\left(x_{1}\right)=\sum_{j=0}^{n} D_{1 j}^{(1)} u_{j}=B, \\
u^{\prime \prime}\left(x_{1}\right) & =\sum_{j=0}^{n} D_{1 j}^{(2)} u_{j}=C,
\end{aligned}
$$

where $\left.D^{(k)}=\left[D_{i j}^{(k)}\right]_{(n+1) \times(n+} 1\right)$,

$$
\begin{aligned}
D_{i j}^{(1)}= \begin{cases}\frac{w_{i}}{x_{i}-x_{j}}, \quad i \neq j, \\
-\sum_{k \neq i} D_{i k}^{(1)}, \quad i=j,\end{cases} \\
D_{i j}^{(2)}= \begin{cases}2\left(D_{i i}^{(1)} D_{i j}^{(1)}-\frac{D_{i j}^{(1)}}{x_{i}-x_{j}}\right), & i \neq j, \\
-\sum_{k \neq i} D_{i k}^{(2)}, & i=j,\end{cases} \\
\mathbf{f}=\left[\begin{array}{ll}
\left.f\left(x_{0}\right), f\left(x_{1}\right), \ldots, f\left(x_{n}\right)\right]^{T} . \\
3\left(D_{i i}^{(2)} D_{i j}^{(1)}-\frac{D_{i j}^{(2)}}{x_{i}-x_{j}}\right), & i \neq j,
\end{array}\right. \\
\mathbf{u}=\left\{\begin{array}{l}
\left.u_{0}, u_{1}, \ldots, u_{n}\right]^{T}, \\
-\sum_{k \neq i} D_{i k}^{(3)},
\end{array}\right.
\end{aligned}
$$

\section{Convergence and Error Analysis}

In this section, we will consider the error problem of equidistant interpolation nodes:

$$
x_{i}=a+\frac{(b-a)}{n} i, \quad i=0,1, \ldots, n .
$$

Let $u(x)$ be the solution of (1); for any $0 \leq d \leq n$, suppose $P\left(x_{i}\right), i=0,1, \ldots, n-d$, to be the barycentric interpolation function at the point $x_{i}, x_{i+1}, \ldots, x_{i+d}$; then, we have $P_{i}\left(x_{k}\right)=f\left(x_{k}\right), k=i, \quad i+1, \ldots, i+d$, and

$$
r(x)=\frac{\sum_{i=0}^{n-d} \lambda_{i}(x) P_{i}(x)}{\sum_{i=0}^{n-d} \lambda_{i}(x)}
$$

where

$$
\lambda_{i}(x)=\frac{(-1)^{i}}{\left(x-x_{i}\right) \cdots\left(x-x_{i+d}\right)} .
$$

Then, the error function is defined as

$$
\begin{gathered}
e(x):=u(x)-P(x)=\left(x-x_{i}\right) \cdots \\
\left(x-x_{i+d}\right)\left[x_{i}, x_{i+1}, \ldots, x_{i+d}, x\right] f
\end{gathered}
$$

and

$$
e(x)=\frac{\sum_{i=0}^{n-d} \lambda_{i}(x)\left(u(x)-P_{i}(x)\right)}{\sum_{i=0}^{n-d} \lambda_{i}(x)}=\frac{A(x)}{B(x)}=O\left(h^{d+1}\right),
$$

where $\quad A(x):=\sum_{i=0}^{n-d}(-1)^{i}\left[x_{i}, \ldots, x_{i+d}, x\right] f$ and $B(x)$ : $=\sum_{i=0}^{n-d} \lambda_{i}(x)$.

Taking the numerical form,

$$
\begin{aligned}
& \sum_{j=0}^{n} u_{j} L_{j}^{\prime \prime}(x)+p \sum_{j=0}^{n} u_{j} L_{j}^{\prime \prime}(x)+q \sum_{j=0}^{n} u_{j} L_{j}^{\prime}(x)+r \\
& \sum_{j=0}^{n} u_{j} L_{j}(x)=f(x),
\end{aligned}
$$

and combining (24) and (1), we get

$$
e^{\prime \prime \prime}(x)+p e^{\prime \prime}(x)+q e^{\prime}(x)+r e(x)=R_{f}(x),
$$

where $R_{f}(x)=f(x)-f\left(x_{k}\right), k=0,1,2, \ldots, n$.

Lemma 1. For e $(x)$ defined in (23), we have

$$
\left\{\begin{array}{l}
|e(x)| \leq C h^{d+1}, \quad u \in C^{d+2}[a, b], \\
\left|e^{\prime}(x)\right| \leq C h^{d}, \quad u \in C^{d+2}[a, b], \\
\left|e^{\prime \prime}(x)\right| \leq C h^{d-1}, \quad u \in C^{d+3}[a, b], \quad d \geq 1, \\
\left|e^{\prime \prime \prime}(x)\right| \leq C h^{d-2}, \quad u \in C^{d+4}[a, b], \quad d \geq 2 .
\end{array}\right.
$$

Let $u(x)$ be the solution of (1) and $u_{n}(x)$ be the numerical solution; then, we have

$$
\begin{gathered}
u_{n}^{\prime \prime}\left(x_{k}\right)+p u_{n}^{\prime \prime}\left(x_{k}\right)+q u_{n}^{\prime}\left(x_{k}\right)+r u_{n}\left(x_{k}\right) \\
\quad=f\left(x_{k}\right), \quad k=0,1,2, \ldots, \ldots n \ldots,
\end{gathered}
$$


and

$$
\lim _{n \longrightarrow \infty} u_{n}(x)=u(x) .
$$

The results can be obtained in [1].

Based on Lemma 1, we can get the following theorem.

Theorem 1. Let $f(x) \in C[a, b], T u(x):=u^{\prime \prime \prime}(x)+p u^{\prime \prime}$ $(x)+q u^{\prime}(x)+r u(x)$, $u_{n}(x): T u_{n}(x)=f(x), u_{n}^{*}(x): T u_{n}^{*}(x)=f^{*}(x)$; then, we have

$$
\left|u_{n}(x)-u_{n}^{*}(x)\right| \leq C h^{d-2}
$$

Proof. Let L: $=D^{(3)}+p D^{(2)}+q D^{(1)}+r I$

$$
=\left[\begin{array}{cccc}
D_{00}^{(3)}+p D_{00}^{(2)}+q D_{00}^{(1)}+r & D_{01}^{(3)}+p D_{01}^{(2)}+q D_{01}^{(1)} & \cdots & D_{0 n}^{(3)}+p D_{0 n}^{(2)}+q D_{0 n}^{(1)} \\
D_{10}^{(3)}+p D_{10}^{(2)}+q D_{10}^{(1)} & D_{11}^{(3)}+p D_{11}^{(2)}+q D_{11}^{(1)}+r & \cdots & D_{1 n}^{(3)}+p D_{1 n}^{(2)}+q D_{1 n}^{(1)} \\
\cdots & \cdots & \cdots & \cdots \\
D_{n 0}^{(3)}+p D_{n 0}^{(2)}+q D_{n 0}^{(1)} & D_{n 1}^{(3)}+p D_{n 1}^{(2)}+q D_{n 1}^{(1)} & \cdots & D_{n n}^{(3)}+p D_{n n}^{(2)}+q D_{n n}^{(1)}+r
\end{array}\right] \text {. }
$$

Add column 2, column 3, . , column $n$ to column 1, and we have

$$
\mathrm{L}=\left[\begin{array}{cccc}
r & D_{01}^{(3)}+p D_{01}^{(2)}+q D_{01}^{(1)} & \cdots & D_{0 n}^{(3)}+p D_{0 n}^{(2)}+q D_{0 n}^{(1)} \\
r & D_{11}^{(3)}+p D_{11}^{(2)}+q D_{11}^{(1)}+r & \cdots & D_{1 n}^{(3)}+p D_{1 n}^{(2)}+q D_{1 n}^{(1)} \\
\cdots & \cdots & \cdots & \cdots \\
r & D_{n 1}^{(3)}+p D_{n 1}^{(2)}+q D_{n 1}^{(1)} & \cdots & D_{n n}^{(3)}+p D_{n n}^{(2)}+q D_{n n}^{(1)}+r
\end{array}\right]
$$

Then, we have $|\mathbf{L}| \neq 0$ with $\quad r \neq 0, \quad u_{n}(x)=\sum_{j=0}^{n} L_{j}$ $(x) f_{j}$, and $u_{n}^{*}(x)=\sum_{j=0}^{n} L_{j}(x) f_{j}^{*}$, where $U_{n}=\left(f\left(x_{0}\right), f\right.$ $\left.\left(x_{1}\right), \ldots, f\left(x_{n}\right)\right)^{T}$ and $U_{n}^{*}=\left(f^{*}\left(x_{0}\right)\right.$, $\left.f^{*}\left(x_{1}\right), \ldots, f^{*}\left(x_{n}\right)\right)^{T}$.

By

$$
U_{n}-U_{n}^{*}=\mathbf{L}^{-1}\left(\mathbf{L} U_{n}-F_{n}^{*}\right),
$$

which means

$$
u_{n}(x)-u_{n}^{*}(x)=\sum M_{j}(x) \operatorname{Te}(x),
$$

where $M_{j}(x)$ is the element of matrix $\mathbf{L}^{-1}$, we have

$$
\left|u_{n}(x)-u_{n}^{*}(x)\right| \leq\left|\sum M_{j}(x)\right||T e(x)| \leq C h^{d-2} .
$$

The proof is completed.

\section{Numerical Example}

As an example, we consider the two-point boundary value problem:

$$
\begin{gathered}
y^{\prime \prime \prime}+y=f(x), \quad-1<x<1, \\
y(-1)=0, y(1)=0, y^{\prime}(-1)=0 .
\end{gathered}
$$

For this problem, we can find a function $f(x)$ such that the analysis solution is

$$
y=\left(1-x^{2}\right)(1+x) e^{\lambda x},
$$

where $\lambda$ is a freely selected parameter.

Substituting (38) into (36), we get

$$
\begin{aligned}
f(x)= & {\left[-6-6 \lambda(1+3 x)+3 \lambda^{2}\left(1-2 x-3 x^{2}\right)\right.} \\
& \left.+\lambda^{3}\left(1+x-x^{2}-x^{3}\right)+\left(1-x^{2}\right)(1+x)\right] e^{\lambda x} .
\end{aligned}
$$

For different values of $d$ and different number of nodes, we can calculate the corresponding relative error and convergence rate; some of the data are shown in Tables 1 and 2.

In Table 1, the convergence rate of equidistant nodes with different $d$ is $O\left(h^{d-2}\right)$; in Table 2, the convergence rate of the Chebyshev point of the second kind with different $d$ is $O\left(h^{d+2}\right), d \geq 2$.

For different values of $\lambda$ and different number of nodes, we can calculate the corresponding relative error; some of the data are shown in Tables 3 and 4.

From Tables 3 and 4 , we can find that, for different values of $\lambda$, the convergence rate can reach $O\left(h^{d+2}\right)(d \geq 2)$ both for equidistant and nonequidistant nodes. 
TABle 1: Errors and convergence rate of the equidistant nodes with different $d$.

\begin{tabular}{|c|c|c|c|c|c|c|c|c|}
\hline \multirow{3}{*}{$n$} & \multicolumn{6}{|c|}{$(\lambda=2)$} & \multirow{2}{*}{\multicolumn{2}{|c|}{$d=5$}} \\
\hline & \multicolumn{2}{|c|}{$d=2$} & \multicolumn{2}{|c|}{$d=3$} & \multicolumn{2}{|c|}{$d=4$} & & \\
\hline & Error & $h^{\alpha}$ & Error & $h^{\alpha}$ & Error & $h^{\alpha}$ & Error & $h^{\alpha}$ \\
\hline 10 & $7.1976 e+00$ & & $4.6412 e+00$ & & $2.4217 e+00$ & & $1.1522 e+00$ & \\
\hline 20 & $3.5555 e+00$ & 1.0175 & $1.2336 e+00$ & 1.9117 & $3.6419 e-01$ & 2.7333 & $9.9594 e-02$ & 3.5322 \\
\hline 40 & $1.4588 e+00$ & 1.2853 & $2.6378 e-01$ & 2.2254 & $4.1431 e-02$ & 3.1359 & $6.0309 e-03$ & 4.0456 \\
\hline 80 & $5.5050 e-01$ & 1.4060 & $5.1045 e-02$ & 2.3695 & $4.1384 e-03$ & 3.3236 & $3.1073 e-04$ & 4.2786 \\
\hline 160 & $2.0058 e-01$ & 1.4566 & $9.4229 e-03$ & 2.4375 & $3.8810 e-04$ & 3.4146 & $1.4799 e-05$ & 4.3921 \\
\hline 320 & $7.1879 e-02$ & 1.4806 & $1.7004 e-03$ & 2.4703 & $3.5301 e-05$ & 3.4586 & $6.7836 e-07$ & 4.4473 \\
\hline 640 & $2.5569 e-02$ & 1.4912 & $3.0357 e-04$ & 2.4858 & $3.1616 e-06$ & 3.4810 & $3.1718 e-08$ & 4.4187 \\
\hline 1280 & $9.0650 e-03$ & 1.4960 & $5.3912 e-05$ & 2.4933 & $2.8666 e-07$ & 3.4632 & $1.2685 e-08$ & 1.3222 \\
\hline
\end{tabular}

TABLE 2: Errors and convergence rate of the Chebyshev point with different $d$.

\begin{tabular}{|c|c|c|c|c|c|c|c|c|}
\hline \multirow{3}{*}{$n$} & \multicolumn{6}{|c|}{$(\lambda=2)$} & \multirow{2}{*}{\multicolumn{2}{|c|}{$d=5$}} \\
\hline & \multicolumn{2}{|c|}{$d=2$} & \multicolumn{2}{|c|}{$d=3$} & \multicolumn{2}{|c|}{$d=4$} & & \\
\hline & Error & $h^{\alpha}$ & Error & $h^{\alpha}$ & Error & $h^{\alpha}$ & Error & $h^{\alpha}$ \\
\hline 10 & $2.4329 e+00$ & & $1.3897 e+00$ & & $2.5216 e-01$ & & $1.1033 e-01$ & \\
\hline 20 & $8.8604 e-01$ & 1.4572 & $1.3680 e-02$ & 6.6665 & $4.5076 e-03$ & 5.8058 & $1.5195 e-03$ & 6.1821 \\
\hline 40 & $1.8242 e-01$ & 2.2801 & $2.8639 e-03$ & 2.2561 & $1.4419 e-04$ & 4.9664 & $7.2659 e-06$ & 7.7082 \\
\hline 80 & $3.2188 e-02$ & 2.5026 & $1.7517 e-04$ & 4.0311 & $3.0543 e-06$ & 5.5610 & $1.0125 e-07$ & 6.1652 \\
\hline 160 & $5.2215 e-03$ & 2.6240 & $8.5892 e-06$ & 4.3501 & $5.9098 e-08$ & 5.6916 & $1.0071 e-06$ & - \\
\hline 320 & $8.0770 e-04$ & 2.6926 & $2.9760 e-07$ & 4.8511 & $1.9760 e-06$ & - & $2.2948 e-05$ & - \\
\hline 640 & $1.2077 e-04$ & 2.7416 & $2.8632 e-06$ & - & $6.856 e-05$ & - & $1.0211 e-03$ & - \\
\hline 1280 & $1.7925 e-05$ & 2.7522 & $7.4861 e-05$ & - & $1.2910 e-02$ & - & $9.6669 e-02$ & - \\
\hline
\end{tabular}

TABLE 3: Errors and convergence rate of the equidistant nodes with different $\lambda$.

\begin{tabular}{|c|c|c|c|c|c|c|c|c|}
\hline \multirow{3}{*}{$n$} & \multicolumn{6}{|c|}{$(d=4)$} & \multirow{2}{*}{\multicolumn{2}{|c|}{$\lambda=20$}} \\
\hline & \multicolumn{2}{|c|}{$\lambda=-5$} & \multicolumn{2}{|c|}{$\lambda=1$} & \multicolumn{2}{|c|}{$\lambda=5$} & & \\
\hline & Error & $h^{\alpha}$ & Error & $h^{\alpha}$ & Error & $h^{\alpha}$ & Error & $h^{\alpha}$ \\
\hline 10 & $3.6809 e+01$ & & $2.2939 e-01$ & & $2.1508 e+02$ & & $3.0238 e+08$ & \\
\hline 20 & $1.3259 e+01$ & 1.4731 & $2.6894 e-02$ & 3.0925 & $6.1838 e+01$ & 1.7983 & $8.0305 e+08$ & - \\
\hline 40 & $2.2319 e+00$ & 2.5707 & $2.7208 e-03$ & 3.3052 & $9.5448 e+00$ & 2.6957 & $6.7632 e+08$ & $2.4778 e-01$ \\
\hline 80 & $2.6875 e-01$ & 3.0539 & $2.5675 e-04$ & 3.4056 & $1.1054 e+00$ & 3.1101 & $1.6623 e+08$ & 2.0246 \\
\hline 160 & $2.7599 e-02$ & 3.2835 & $2.3416 e-05$ & 3.4548 & $1.1147 e-01$ & 3.3098 & $2.4028 e+07$ & 2.7904 \\
\hline 320 & $2.6249 e-03$ & 3.3943 & $2.1009 e-06$ & 3.4784 & $1.0509 e-02$ & 3.4070 & $2.7001 e+06$ & 3.1536 \\
\hline
\end{tabular}

TABle 4: Errors and convergence rate of the Chebyshev point with different $\lambda$.

\begin{tabular}{|c|c|c|c|c|c|c|c|c|}
\hline \multirow{3}{*}{$n$} & \multicolumn{6}{|c|}{$(d=4)$} & \multirow{2}{*}{\multicolumn{2}{|c|}{$\lambda=20$}} \\
\hline & \multicolumn{2}{|c|}{$\lambda=-5$} & \multicolumn{2}{|c|}{$\lambda=1$} & \multicolumn{2}{|c|}{$\lambda=5$} & & \\
\hline & Error & $h^{\alpha}$ & Error & $h^{\alpha}$ & Error & $h^{\alpha}$ & Error & $h^{\alpha}$ \\
\hline 10 & $1.0975 e+01$ & & $1.8992 e-02$ & & $4.4245 e+01$ & & $1.3431 \mathrm{e} \_01$ & \\
\hline 20 & $1.5012 e-01$ & 6.1919 & $2.6112 e-04$ & 6.1845 & $1.3603 e+00$ & 5.0235 & $2.5740 e+08$ & 2.3835 \\
\hline 40 & $5.5289 e-03$ & 4.7630 & $8.0762 e-06$ & 5.0149 & $4.7712 e-02$ & 4.8334 & $1.4800 e+07$ & 4.1204 \\
\hline 80 & $1.1729 e-04$ & 5.5589 & $1.6777 e-07$ & 5.5891 & $1.0350 e-03$ & 5.5267 & $3.5715 e+05$ & 5.3729 \\
\hline 160 & $2.2528 e-06$ & 5.7022 & $1.8130 e-08$ & 3.2100 & $2.3528 e-05$ & 5.4591 & $8.3139 e+03$ & 5.4249 \\
\hline 320 & $1.3043 e-05$ & - & $2.5236 e-06$ & - & $2.4682 e-06$ & 3.2529 & $2.1982 e+02$ & 5.2411 \\
\hline
\end{tabular}




\section{Conclusion}

In this paper, the barycentric rational collocation method for solving third-order two-point boundary value equations is presented, and the error function of the convergence rate $O\left(h^{d-2}\right)$ is also obtained. For the constant coefficient and variable coefficient of two-point boundary value equations, numerical results show that the convergence rate can reach $O\left(h^{d-2}\right)$ for the equidistant nodes and Chebyshev point of the second kind with $d \geq 2$, so the barycentric rational collocation method is an effective method. Compared with other methods, the advantage of this method is that the matrix equation can be easily obtained, the program is simple, and high computational accuracy can be obtained by using a few points.

\section{Data Availability}

The data that support the findings of this study are available from the corresponding author upon reasonable request.

\section{Conflicts of Interest}

The authors declare that there are no conflicts of interest regarding the publication of this paper.

\section{Acknowledgments}

The support from the Industry-University Cooperation Collaborative Education Project (201801123024) is gratefully acknowledged.

\section{References}

[1] J.-P. Berrut, M. S. Floater, and G. Klein, "Convergence rates of derivatives of a family of barycentric rational interpolants," Applied Numerical Mathematics, vol. 61, no. 9, pp. 989-1000, 2011.

[2] J.-P. Berrut, S. A. Hosseini, and G. Klein, "The linear barycentric rational quadrature method for Volterra integral equations," SIAM Journal on Scientific Computing, vol. 36, no. 1, pp. 105-123, 2014.

[3] J.-P. Berrut and G. Klein, "Recent advances in linear barycentric rational interpolation," Journal of Computational and Applied Mathematics, vol. 259, pp. 95-107, 2014.

[4] A. Gillette, A. Rand, and C. Bajaj, "Error estimates for generalized barycentric interpolation," Advances in Computational Mathematics, vol. 37, no. 3, pp. 417-439, 2012.

[5] G. Klein and J.-P. Berrut, "Linear rational finite differences from derivatives of barycentric rational interpolants," SIAM Journal on Numerical Analysis, vol. 50, no. 2, pp. 643-656, 2012.

[6] G. Klein and J.-P. Berrut, "Linear barycentric rational quadrature," BIT Numerical Mathematics, vol. 52, no. 2, pp. 407-424, 2012.

[7] M. S. Floater and K. Hormann, "Barycentric rational interpolation with no poles and high rates of approximation," Numerische Mathematik, vol. 107, no. 2, pp. 315-331, 2007.

[8] J. Li and Y. Cheng, "Linear barycentric rational collocation method for solving second-order Volterra integro-differential equation," Computational and Applied Mathematics, vol. 39, no. 2, pp. A1936-A1960, 2020.
[9] M. Li and C. Huang, "The linear barycentric rational quadrature method for auto-convolution Volterra integral equations," Journal of Scientific Computing, vol. 78, no. 1, pp. 549-564, 2019.

[10] A. Abdi, J.-P. Berrut, and S. A. Hosseini, "The linear barycentric rational method for a class of Delay Volterra integrodifferential equations," Journal of Scientific Computing, vol. 75, no. 3, pp. 1757-1775, 2018.

[11] J. Li and Y. Cheng, "Numerical solution of Volterra integrodifferential equations with linear barycentric rational method," International Journal of Applied and Computational Mathematics, vol. 6, p. 137, 2020.

[12] Z. Wang, L. Zhang, Z. Xu, and J. Li, "Barycentric interpolation collocation method based on mixed displacement-stress formulation for solving plane elastic problems," Chinese Journal of Applied Mechanics, vol. 35, no. 2, pp. 304-309, 2018.

[13] Z. Wang and S. Li, Barycentric Interpolation Collocation Method for Nonlinear Problems, National Defense Industry Press, Beijing, China, 2015.

[14] J. Li and Y. Cheng, "Linear barycentric rational collocation method for solving heat conduction equation," Numerical Methods for Partial Differential Equations, vol. 37, no. 1, pp. 533-545, 2021.

[15] E. Cirillo and K. Hormann, "On the Lebesgue constant of barycentric rational Hermite interpolants at equidistant nodes," Journal of Computational and Applied Mathematics, vol. 349, pp. 292-301, 2019.

[16] Z. Wang, Z. Xu, and J. Li, "Mixed barycentric interpolation collocation method of displacement-pressure for incompressible plane elastic problems," Chinese Journal of Applied Mechanics, vol. 35, no. 3, pp. 195-201, 2018.

[17] J. Li and Y. Cheng, "Barycentric rational method for solving biharmonic equation by depression of order," Numerical Methods for Partial Differential Equations, pp. 1-15, 2020.

[18] E. Ugurlu, "Some singular third-order boundary value problems," Mathematical Methods in the Applied Sciences, vol. 43, pp. 2202-2215, 2020.

[19] X. Feng, H. Feng, and H. Tan, "Existence and iteration of positive solutions for third-order Sturm-Liouville boundary value problems with p-Laplacian," Applied Mathematics and Computation, vol. 266, pp. 634-641, 2015.

[20] M. Abushammala, S. A. Khuri, and A. Sayfy, "A novel fixed point iteration method for the solution of third order boundary value problems," Applied Mathematics and Computation, vol. 271, pp. 131-141, 2015. 